\title{
Deciphering the Corelease of Glutamate from Dopaminergic Terminals Derived from the Ventral Tegmental Area
}

\author{
Xing-Long Gu \\ Unit of Transgenesis, Laboratory of Neurogenetics, National Institute on Aging, National Institutes of Health, Bethesda, Maryland 20892 \\ Review of Stuber et al.
}

The ventral tegmental area (VTA) is located close to the midline on the floor of the midbrain. VTA neurons project densely and largely ipsilaterally to the ventromedial striatum, primarily the nucleus accumbens (NAc) core and shell. The neuronal circuit between VTA and NAc is involved in the acquisition and expression of learned appetitive behavior and in drug addiction. Approximately $60 \%$ of VTA neurons are dopaminergic and abnormal dopaminergic signaling in the NAc is thought to be a critical component in several neuropsychiatric disorders.

Increasing evidence indicates that some dopaminergic neurons may also use glutamate as a neurotransmitter in the striatum (Descarries et al., 2008). Selective stimulation and intracellular recording of synaptic responses in autaptic cultures of dopaminergic neurons from VTA suggested that dopamine and glutamate were released by the same neuron (Sulzer et al., 1998). In cocultures of dopaminergic and accumbens neurons, stimulation of dopaminergic neurons evoked glutamate-mediated excitatory synaptic responses in accumbens neurons (Joyce and Rayport, 2000). Additionally, release of glu-

Received July 21, 2010; revised Aug. 25, 2010; accepted Aug. 25, 2010.

This work was supported by the postdoctoral fellowship from National Institute on Aging, National Institutes of Health. I thank Dr. David Lovinger for helpful discussion and manuscript editing.

Correspondence should be addressed to Xing-Long Gu, 35 Convent Drive, Building 35, Room 1A1006, Bethesda, MD 20892. E-mail: xlgu2007@ gmail.com.

DOI:10.1523/JNEUROSCI.3802-10.2010

Copyright $\odot 2010$ the authors $\quad$ 0270-6474/10/3013549-03\$15.00/0 tamate by VTA dopaminergic neurons was suggested by the presence of vesicular glutamate transporter 2 (VGluT2), a marker of glutamatergic neurons (Dal Bo et al., 2004). In mesoaccumbens slice preparations, glutamate-mediated excitatory responses were detected in postsynaptic accumbens neurons after focal extracellular stimulation of dopaminergic neurons in the VTA (Chuhma et al., 2004; Hnasko et al., 2010). However, whether or not glutamate is released from the terminals of dopaminergic neurons in vivo is controversial because extracellular stimulation might excite nondopaminergic neurons in VTA that express VGluT2 and release glutamate in NAc (Kawano et al., 2006; Mendez et al., 2008; Hnasko et al., 2010).

In a paper recently published in the The Journal of Neuroscience by Stuber et al. (2010), the light-activated cation channel channelrhodopsin-2 (ChR2) was selectively expressed in midbrain dopaminergic neurons and its fibers in striatum. Subsequent optical stimulation of dopaminergic terminals in NAc evoked glutamate-mediated postsynaptic responses in medium spiny neurons (MSNs), and these responses were abolished by conditional knock-out of VGluT2 in dopaminergic neurons. Similar results were also reported recently by Tecuapetla et al. (2010).

Specific expression of ChR2-mcherry in midbrain dopaminergic neurons was achieved by injecting Cre-inducible adenoassociated virus vectors carrying a gene encoding ChR2 into the VTA of adult mice that expressed Cre under the control of the dopamine transporter promoter (Stuber et al., 2010, their Fig. 1). Lightevoked stimulation of labeled dopaminergic terminals resulted in AMPA receptormediated EPSCs in all recorded MSNs in the NAc shell (Stuber et al., 2010, their Figs. 2, 3), suggesting that glutamate was released from the dopaminergic terminals. In agreement with this, Tecuapetla et al. (2010) found that stimulation of dopaminergic terminals activated both AMPA and NMDA receptors in all recorded MSNs. These two studies provide clear and straightforward evidence that dopaminergic neurons release glutamate in the NAc. In addition, conditional knock-out of VGluT2 in dopaminergic neurons abolished EPSCs evoked by optical stimulation (Stuber et al., 2010, their Figs. 2, 3), strongly suggesting that glutamate mediates the EPSCs evoked by stimulation of dopaminergic terminals.

Previous electron microscopic (EM) data suggested that both synaptic (boutons) and nonsynaptic (varicosities) release sites were present in dopaminergic axon branches in the striatum (Descarries et al., 2008). The dopaminergic varicosities presumably exhibit no obvious presynaptic and postsynaptic membrane or classic active zone (Fig. $1 B, D, F$ ), but dopamine released from varicosities might diffuse in the extracellular space to reach dopamine receptors (Descarries et al., 2008). Given two types of release sites and the fact that glutamate and dopamine could be packaged into the same or different vesicles, eight patterns of glutamate 
and dopamine release are theoretically possible in the striatum (Fig. 1). Optical stimulation combined with electrophysiology is unable to provide clues about which pattern of glutamate release occurs in the NAc. For example, whether dopaminergic VTA neurons have some boutons or varicosities that only release glutamate in the NAc is still an open question (Fig. 1G,H). However, it is clear that synaptic boutons release both dopamine and glutamate (Fig. 1C,E), based on the rapid postsynaptic response to light stimulation and morphological evidence from previous EM studies (Descarries et al., 2008; Stuber et al., 2010). Tyrosine hydroxylase is coexpressed with VGluT2 in presynaptic boutons visualized by EM, indicating that dopamine and glutamate are likely present in the same presynaptic terminals. Furthermore, vesicular monoamine transporter 2 (VMAT2) can be coimmunoprecipitated with VGluT2 from synaptic vesicle preparations from rat striatum, suggesting that VMAT2 and VGluT2 physically interact and/or are located on the same presynaptic vesicle membranes. Therefore, dopamine and glutamate are very likely to be packaged into the same synaptic vesicle by VMAT2 and VGluT2, respectively (Fig. 1C) (Hnasko et al., 2010). Moreover, synaptic vesicular coentry of dopamine and glutamate promotes monoamine storage by increasing the $\mathrm{pH}$ gradient that drives vesicular monoamine transport (Hnasko et al., 2010). However, it is not clear whether dopamine and glutamate are also packaged into separate synaptic vesicles (Fig. $1 E$ ). This question can be answered using electron microscopy to examine the double immunolabeling of VMAT2 and VGluT2 in striatum tissue.

Stuber et al. (2010) found that corelease of dopamine and glutamate was restricted to the NAc shell. Glutamatemediated current was not detected in the dorsal striatum after optical stimulation, although light-evoked dopamine release was detected voltammetrically in both the dorsal striatum and NAc shell (Stuber et al., 2010, their Fig. 4). This suggests that VTA dopaminergic terminals in dorsal striatum do not release glutamate, or release minimal glutamate that is unable to evoke detectable EPSCs. Additionally, this result also suggests that substantia nigra pars compacta $(\mathrm{SNc})$ dopaminergic neurons do not release glutamate, because dopaminergic terminals in dorsal striatum are largely derived from SNc dopaminergic neurons, which were infected with virus along with VTA dopaminergic

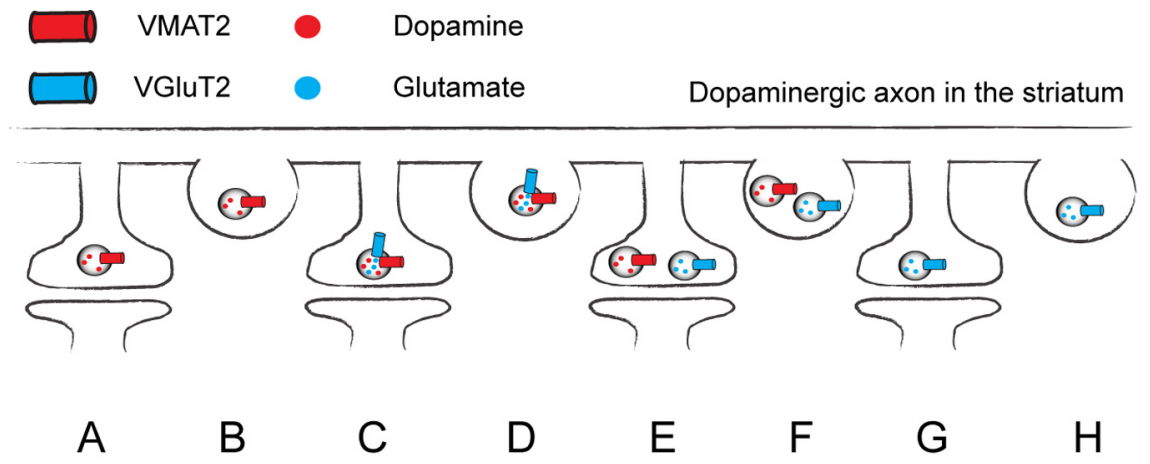

Figure 1. Schematic representation of possibilities of synaptic and nonsynaptic dopaminergic axon terminals containing either or both dopamine and glutamate. The synaptic terminals display a classical active zone, postsynaptic density, and synaptic cleft $(\boldsymbol{A}, \boldsymbol{C}, \boldsymbol{E}$, and $\boldsymbol{G})$. The nonsynaptic terminals (varicosities) show the lack of presynaptic and postsynaptic specializations $(\boldsymbol{B}, \boldsymbol{D}$, $\boldsymbol{F}$, and $\boldsymbol{H})$.

neurons (Stuber et al., 2010, their Fig. 1). It is consistent with previous study that SNc dopaminergic neurons lack VGluT2 and therefore are unlikely to release glutamate (Hnasko et al., 2010).

It is surprising that the clearance of dopamine (via uptake by the dopamine transporter) after release in the NAc shell is much slower than that in the dorsal striatum, as suggested by the fact that the decay time of the dopamine transient is much longer in the NAc shell (Stuber et al., 2010, their Fig. 4). Given that VGluT2 expression is correlated with the slowed dopamine uptake, it would be interesting to know whether glutamate-releasing dopaminergic terminals express less dopamine transporter, which decreases the rate of dopamine reuptake. It is also possible that released glutamate activates glutamate receptors and consequently alters local ion concentration gradient, which is the driving force for dopamine transporter-mediated dopamine uptake. To study the role of VGluT2 and glutamate in dopamine reuptake, measurement of optically evoked dopamine release in the striatum could be performed in the VGluT2 conditional knock-out and wild-type control mice with or without the presence of glutamate receptor antagonists.

From Stuber et al. (2010) and previous studies (Descarries et al., 2008), it is clear that dopamine and glutamate are packaged into the same synaptic vesicles and released from the dopaminergic terminals originating from a sublet of dopaminergic neurons in the VTA that project to the NAc shell. Although presence of glutamate in the dopaminergic vesicles promotes dopamine sequestration into presynaptic vesicles (Hnasko et al., 2010), the postsynaptic function of glutamate release from VTA dopaminergic neurons remains unclear. It is not clear from Stu- ber et al. (2010) whether glutamate from dopaminergic boutons or varicosities can evoke action potentials in MSNs of the NAc, or whether glutamate-evoked EPSCs merely boost the excitability by depolarizing the MSNs to a membrane potential close to spike threshold. The membrane potentials of MSNs move between a down state, where neurons are slightly hyperpolarized and do not generate action potentials, and an up state, where neurons sit at a membrane potential close to the threshold for spike generation in response to temporally coherent, convergent-excitatory synaptic input from the cortex (Wilson and Kawaguchi, 1996). Thus, glutamatergic excitatory input from VTA dopaminergic neurons might work synergistically with cortical excitatory input to modulate MSNs activities in striatum. Consistent with this hypothesis, Tecuapetla et al. (2010) found that glutamate-evoked EPSCs elicited action potentials at membrane potentials close to the typical MSN up state in five of seven recorded MSNs after a single optical stimulation.

The effect of glutamate corelease at dopaminergic synapses is likely to depend on what dopamine receptor is present. Most MSNs in NAc express either D1 or D2 receptors, depending on the projection target (D1 receptors in striatonigral neurons, D2 receptors in striatopallidal neurons) (Humphries and Prescott, 2010), but accumulating evidence indicates that some NAc neurons express both D1 and D2 receptors. Several lines of evidence suggest that D1 receptor signaling enhances, whereas D2 receptor signaling inhibits, dendritic excitability and glutamatergic signaling, at least in dorsal striatum. Therefore, if D1 receptor is present at the postsynaptic site of synapses at which glutamate and dopamine are coreleased, glutamate and 
dopamine would synergistically regulate the excitability of MSNs. However, it is also possible that glutamate and dopamine modulate the excitability of MSNs in opposite ways by corelease at D2 receptorexpressing synapses, to increase precision. Further functional and morphological studies are needed to address these possibilities.

In conclusion, Stuber et al. provide definitive physiological evidence for the corelease of glutamate from dopaminergic terminals derived from VTA in the NAc. Future studies will most likely focus on the properties as well as the pathophysiological functions of this subgroup of synapses that corelease dopamine and glutamate in the NAc.

\section{References}

Chuhma N, Zhang H, Masson J, Zhuang X, Sulzer D, Hen R, Rayport S (2004) Dopamine neurons mediate a fast excitatory signal via their glutamatergic synapses. J Neurosci 24:972-981.
Dal Bo G, St-Gelais F, Danik M, Williams S, Cotton M, Trudeau LE (2004) Dopamine neurons in culture express VGLUT2 explaining their capacity to release glutamate at synapses in addition to dopamine. J Neurochem 88:1398-1405.

Descarries L, Bérubé-Carrière N, Riad M, Bo GD, Mendez JA, Trudeau LE (2008) Glutamate in dopamine neurons: synaptic versus diffuse transmission. Brain Res Rev 58:290-302.

Hnasko TS, Chuhma N, Zhang H, Goh GY, Sulzer D, Palmiter RD, Rayport S, Edwards RH (2010) Vesicular glutamate transport promotes dopamine storage and glutamate corelease in vivo. Neuron 65:643-656.

Humphries MD, Prescott TJ (2010) The ventral basal ganglia, a selection mechanism at the crossroads of space, strategy, and reward. Prog Neurobiol 90:385-417.

Joyce MP, Rayport S (2000) Mesoaccumbens dopamine neuron synapses reconstructed in vitro are glutamatergic. Neuroscience 99:445-456.

Kawano M, Kawasaki A, Sakata-Haga H, Fukui Y, Kawano H, Nogami H, Hisano S (2006) Particular subpopulations of midbrain and hypothalamic dopamine neurons express vesicular glutamate transporter 2 in the rat brain J Comp Neurol 498:581-592.

Mendez JA, Bourque MJ, Dal Bo G, Bourdeau ML, Danik M, Williams S, Lacaille JC, Trudeau LE (2008) Developmental and target-dependent regulation of vesicular glutamate transporter expression by dopamine neurons. J Neurosci 28:6309-6318.

Stuber GD, Hnasko TS, Britt JP, Edwards RH, Bonci A (2010) Dopaminergic terminals in the nucleus accumbens but not the dorsal striatum corelease glutamate. J Neurosci 30:8229-8233.

Sulzer D, Joyce MP, Lin L, Geldwert D, Haber SN, Hattori T, Rayport S (1998) Dopamine neurons make glutamatergic synapses in vitro. J Neurosci 18:4588-4602.

Tecuapetla F, Patel JC, Xenias H, English D, Tadros I, Shah F, Berlin J, Deisseroth K, Rice ME, Tepper JM, Koos T (2010) Glutamatergic signaling by mesolimbic dopamine neurons in the nucleus accumbens. J Neurosci 30:7105-7110.

Wilson CJ, Kawaguchi Y (1996) The origins of two-state spontaneous membrane potential fluctuations of neostriatal spiny neurons. J Neurosci 16:2397-2410. 